\title{
The mossy fiber bouton: the "common" or the "unique" synapse?
}

\author{
Astrid Rollenhagen' and Joachim H. R. Lübke ${ }^{1,2 *}$ \\ 1 Institute of Neuroscience and Medicine INM-2, Research Centre Jülich, Jülich, Germany \\ 2 Department of Psychiatry and Psychotherapy, Medical Faculty, RWTH Aachen University, Jülich-Aachen Research Alliance - Translational Brain Medicine, Aachen, \\ Germany
}

\section{Edited by:}

Laszlo Acsady, Institute of

Experimental Medicine, Hungary

Reviewed by:

Janos Szabadics, University of Szeged, Hungary

David Amaral, University of California at Davis, USA

Gianmaria Maccaferri, Northwestern

University, USA

\section{${ }^{*}$ Correspondence:}

Joachim H. R. Lübke, Institute of Neuroscience and Medicine INM-2, Research Centre Jülich, D-52425

Jülich, Germany.

e-mail: j.luebke@fz-juelich.de
Synapses are the key elements for signal processing and plasticity in the brain. They are composed of nearly the same structural subelements, an apposition zone including a pre-and postsynaptic density, a cleft and a pool of vesicles. It is, however, their actual composition that determines their different behavior in synaptic transmission and plasticity. Here, we describe and discuss the structural factors underlying the unique functional properties of the hippocampal mossy fiber (MF) synapse. Two membrane specializations, active zones (AZs; transmitter release sites), and puncta adherentia (PA), putative adhesion complexes were found. On average, individual boutons had $20 \mathrm{AZs}$ with a mean surface area of $0.1 \mu \mathrm{m}^{2}$ and a short distance of $0.45 \mu \mathrm{m}$ between individual AZs. Mossy fiber boutons (MFBs) and their target structures were isolated from each other by astrocytes, but fine glial processes never reached the AZs. Therefore, two structural factors are likely to promote synaptic cross-talk: the short distance and the absence of fine glial processes between individual AZs. Thus, synaptic crosstalk may contribute to the high efficacy of hippocampal MF synapses. On average, an adult bouton contained $\sim 16,000$ synaptic vesicles; -600 vesicles were located within $60 \mathrm{~nm}$ from the AZ, 4000 between $60 \mathrm{~nm}$ and $200 \mathrm{~nm}$, and the remaining beyond $200 \mathrm{~nm}$, suggesting large readily releasable, recycling, and reserve pools. Thus, the size of the three pools together with the number and distribution of AZs underlie the unique extent of synaptic efficacy and plasticity of the hippocampal MF synapse.

Keywords: mossy fiber-CA3 pyramidal cell synapse, electron microscopy, 3D-reconstructions, quantitative analysis, actives zones, different pools of synaptic vesicles, synaptic transmission and plasticity, synaptic crosstalk

\section{INTRODUCTION}

Over the last 50 years synapses have been looked at from different viewpoints resulting in numerous original publications and summarized in several reviews and textbooks. Nevertheless, a detailed description of their structural geometry, in particular their quantitative morphology, is still limited to a relatively small number of synapses. Such detailed descriptions are, however, required to understand and link structural and functional components of the signal cascades underlying synaptic transmission and plasticity. Using high end electron microscopy and related techniques that allow subcellular and even molecular resolution it became possible to unravel and quantify the structural subelements of synapses providing important constrains that can be used for realistic numerical simulations of those parameters of synaptic transmission that are still inaccessible to experiment.

Only recently the structural geometry of a synapse embedded in a higher cortical microcircuit, the hippocampal mossy fiber bouton (MFB)-CA3 pyramidal cell synapse, has been described in great detail (Rollenhagen et al., 2007a). In particular, those structural subelements relevant for synaptic transmission and plasticity at the MFB-CA3 pyramidal cell synapse have been investigated using computer-assisted 3D-reconstructions of serial electron microscopic images and subsequent quantitative analysis based on serial ultrathin sections through individual MFBs.
It is widely accepted that the hippocampus is involved in learning and memory function, in particular that mossy fiber (MF) synapses play a key role in processing, storage and recall of spatial information in the hippocampal network (Lisman, 1999; for review see Nicoll and Schmitz, 2005; Bischofberger et al., 2006). Furthermore, the MF system may play a critical role in pattern completion, pattern separation and storage of sequences of events (for review see Bischofberger et al., 2006).

Within the hippocampal formation the MF system is a structurally highly complex connecting the entorhinal cortex via the MF axons of the dentate gyrus granule cells with CA3 pyramidal cells and GABAergic interneurons in the hilar region and the stratum lucidum of the CA3 subregion (for review see Frotscher et al., 2006). It is known that MF axons exhibit three morphologically distinct presynaptic specializations: large 'giant' boutons that are thought to represent the main bodies of MFs (Galimberti et al., 2006), small en passant boutons, and filopodial extensions emerging from the MFBs (Amaral and Dent, 1981). Large MFBs innervate spiny excrescences of CA3 pyramidal neurons (Blackstad and Kjaerheim, 1961; Hamlyn, 1962; Rollenhagen et al., 2007a) and hilar mossy cells (Frotscher et al., 1994). In contrast, the filopodial extensions are thought to contact exclusively the dendritic domain of different GABAergic interneurons in the hilus and stratum lucidum (Acsády et al., 1998; Szabadics and Soltesz, 2009), although large MFBs or 
main bodies also directly contact the dendritic shafts of GABAergic interneurons (for review see Frotscher et al., 2006). A single granule cell axon gives rise to $\sim 15$ large MFBs in the CA3 region, and a single CA3 pyramidal neuron receives input from $\sim 50 \mathrm{MF}$ axons (Amaral et al., 1990). Thus, the morphological properties of the MF synapse are consistent with the view that it forms a powerful, but sparse synaptic connection.

Hippocampal MF synapses show several unique transmission and plasticity properties when compared with other CNS synapses. MF synapses are powerful, generating large postsynaptic currents and potentials in both CA3 pyramidal neurons and GABAergic interneurons (Maccaferri et al., 1998; Henze et al., 2002a; Lawrence et al., 2004). Synaptic plasticity at the MF-CA3 pyramidal cell synapses is unique for several reasons, including a low basal release probability due to the tonic activation of $A_{1}$ adenosine receptors (Moore et al., 2003). Furthermore, MF synapses can undergo substantial changes in synaptic strength and efficacy, exhibiting marked paired pulse- and frequency facilitation, post-tetanic- and NMDA-receptor independent long-term potentiation (LTP; Salin et al., 1996; Kobayashi and Poo, 2004; Engel and Jonas, 2005; Alle and Geiger, 2006; Alle et al., 2009; for review see Nicoll and Schmitz, 2005). Finally, the properties of MF transmission are highly compartmentalized. At MFB-GABAergic interneuron synapses, the extent of synaptic plasticity is markedly smaller when compared with the MFB-CA3 pyramidal neuron synapses (Tóth et al., 2000; Szabadics and Soltesz, 2009). MF inputs to GABAergic interneurons in the stratum lucidum via the filopodial extensions undergo severe long-term depression whereas MF-CA3 pyramidal cell synapses show long-term potentiation triggered by compartimentelized $\mathrm{Ca}^{2+}$ channels. This difference in synaptic plasticity indicate that neighboring, functionally divergent presynaptic elements along the same axon serve as autonomous computational units capable of modifying release independently (Pelkey and McBain, 2005; Pelkey et al., 2006).
Thus, the high synaptic efficacy and plasticity at the MFB-CA3 pyramidal neuron synapses may be partially a result of the organization of the pool of synaptic vesicles in the large MFBs.

Here, we summarize and discuss these findings that underlie the unique functional properties of the hippocampal MF-CA3 pyramidal cell synapse. We demonstrate that several structural features of hippocampal MFBs differ fundamentally from the calyx of Heldprincipal neuron synapse in the medial nucleus of the trapezoid body (MNTB, Rowland et al., 2000; Sätzler et al., 2002; Wimmer et al., 2006) and other previously investigated central presynaptic terminals (climbing and parallel fiber synapse: Xu-Friedman et al., 2001; endbulb of Held: Nicol and Walmsley, 2002; cerebellar mossy fiber synapse: Xu-Friedman and Regehr, 2003; retinal ribbon synapse: Sikora et al., 2005; tom Dieck and Brandstätter, 2006; Midorikawa et al., 2007; GABAergic nigrothalamic terminals: Bodor et al., 2008; input synapses from the nucleus reticularis and anterior pretectal nucleus to the posterior thalamic nucleus: Wanaverbecq et al., 2008; for review see Rollenhagen and Lübke, 2006). Thus, the MFB, with respect to its structural features, can be regarded as a highly specialized, and therefore unique synapse perfectly suited for its function within the hippocampal network.

\section{GENERAL DESCRIPTION OF THE GEOMETRY OF THE MFB}

The axons of granule cells in the dentate gyrus constitute a massive fiber bundle, the MFs that establish large, highly specialized en passant boutons or end terminals (Figure 1A) with proximal dendritic segments predominantly on spiny (thorny) excrescences (Figures 1A and 2A) of excitatory CA3 pyramidal cells and various GABAergic interneurons in the hilus and stratum lucidum (Acsády et al., 1998; Szabadics and Soltesz, 2009). Dendrites of CA3 pyramidal neurons receive dense synaptic input from multiple MFBs (Rollenhagen et al., 2007a); in turn, individual MFBs contact several dendritic segments of the same (Chicurel and Harris, 1992) or different pyramidal neurons (Galimberti et al.,

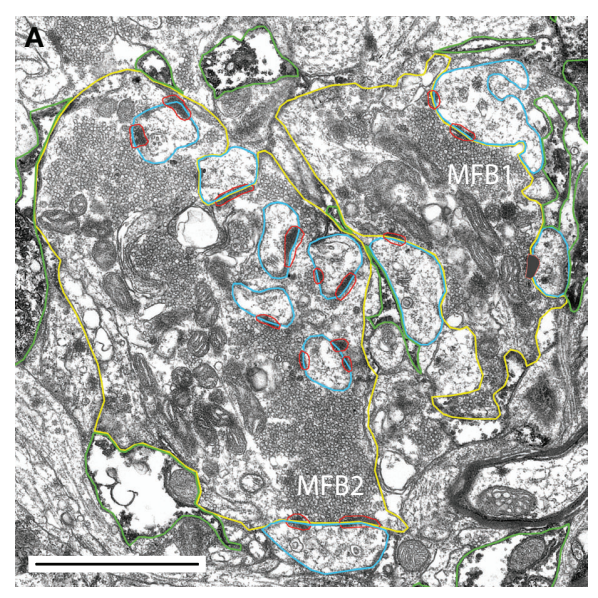

FIGURE 1 |The hippocampal mossy fiber bouton. (A) Low power electron microscopic image of two adjacent MFBs (MFB1, MFB2) taken from an adult rat. Both MFBs outlined in yellow terminate on different dendritic segments, but preferentially on spiny excrescences (blue contours). AZs are given in red and astrocytic profiles (dark diaminobenzidine reaction product) in green. Scale bar: $2.5 \mu \mathrm{m}$. (B) Glial coverage of an individual MFB showing the distribution of

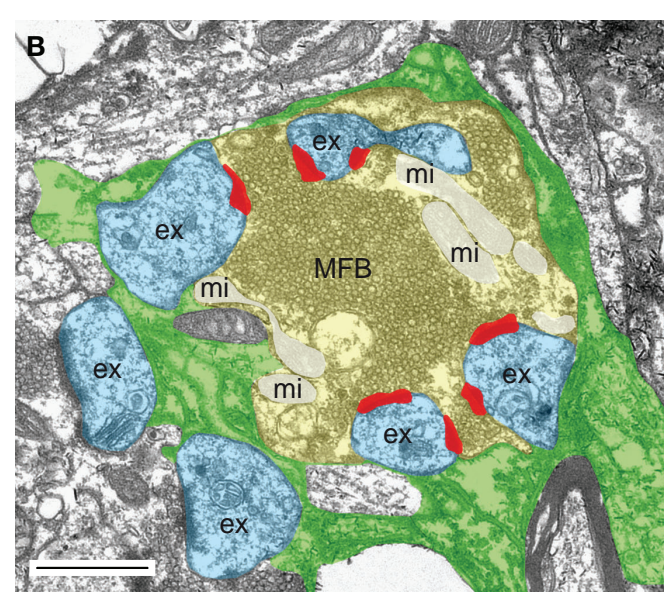

astrocytic processes (highlighted in green) as revealed by glutamine synthetase pre-embedding immunohistochemistry. The surface of an individual MFB (in light yellow), its postsynaptic spiny excrescences (ex; in light blue) was surrounded by several astrocytic processes (in green). Note, that the fine glial processes do not reach the AZs (in red) and the synaptic cleft. The mitochondria (mi) within the MFB are highlighted in white. Scale bar: $1 \mu \mathrm{m}$. 
2006). The density of MFBs terminating on dendritic segments of CA3 pyramidal cells in the stratum lucidum was found to be remarkably high.

Mossy fiber boutons were highly variable in shape and size ranging from 2 to $5 \mu \mathrm{m}$ in cross diameter, from 40 to $110 \mu \mathrm{m}^{2}$ in surface area and from 2 to $13 \mu \mathrm{m}^{3}$ in volume (Table 1 ).

In addition, small caliber boutons ranging from 8.2 and $13.9 \mu \mathrm{m}^{2}$ in surface area and with a volume of less than $1 \mu \mathrm{m}^{3}$ were observed. However, these small boutons may either resemble those described as satellites (Galimberti et al., 2006) or could emerge from filopodia (Acsády et al., 1998). On average, two to four filopodial extensions of various shape and length $(1-5 \mu \mathrm{m})$ emerged from the main body of the bouton, sometimes terminating abruptly or in an endbulb-like structure. These endbulbs often contained a single active zone (AZ).
The main bodies or larger MFBscontained numerous mitochondria of various shape and size (33-57) that either formed cluster-like arrangements or constituted row-like assemblies (Figure 2C). Mitochondria contributed between $4 \%$ and $12 \%$ to the total volume of individual boutons and act not only as internal calcium stores (Rizzuto et al., 2000), but may also regulate internal $\mathrm{Ca}^{2+}$ levels in the terminal. Furthermore, mitochondria may be involved in the mobilization of synaptic vesicles from the reserve pool (Verstreken et al., 2005). In MFBs, mitochondria were seen to be associated with the pool of synaptic vesicles (Figure 2C) suggesting a role for these structures in synaptic vesicle trafficking. However, they were not organized in mitochondria-associated complexes (Rowland et al., 2000) or donut-like assemblies (Wimmer et al., 2006) as described for the calyx of Held nerve terminal. Interestingly, most of the MFBs investigated were penetrated and crossed by other axons of

Table 1 | Quantitative analysis of structural elements of adult MFBs.

\begin{tabular}{|c|c|c|c|c|c|c|c|c|c|}
\hline \multicolumn{3}{|c|}{ Bouton } & \multicolumn{5}{|c|}{ Active zones } & \multicolumn{2}{|c|}{ Cleft width } \\
\hline 1 & 13.03 & 82.19 & 34 & $0.07 \pm 0.05$ & 2.26 & 7.2 & $0.32 \pm 0.09$ & $28.11 \pm 3.26$ & $19.62 \pm 2.63$ \\
\hline 3 & 5.42 & 50.55 & 14 & $0.14 \pm 0.13$ & 2.01 & 9.5 & $0.52 \pm 0.14$ & $29.46 \pm 5.22$ & $20.25 \pm 2.58$ \\
\hline 7 & 2.35 & 32.37 & 7 & $0.15 \pm 0.08$ & 1.07 & 9.4 & $0.47 \pm 0.27$ & $29.81 \pm 3.09$ & $20.48 \pm 0.44$ \\
\hline Mean & 8.24 & 58.50 & 18.3 & $0.11 \pm 0.07$ & 2.10 & 9.7 & $0.48 \pm 0.19$ & $26.78 \pm 1.87$ & $20.25 \pm 1.21$ \\
\hline $\mathrm{CV}$ & 0.63 & 0.37 & & 0.64 & 0.39 & 0.20 & 0.40 & 0.07 & 0.06 \\
\hline
\end{tabular}

Summary of structural parameters relevant for synaptic transmission that have been extracted from the detailed 3-D reconstructions of MFBs. The means and coefficient of variations (CVs) given in this table are averages per bouton (modified from Table 1 in Rollenhagen et al., 2007a).

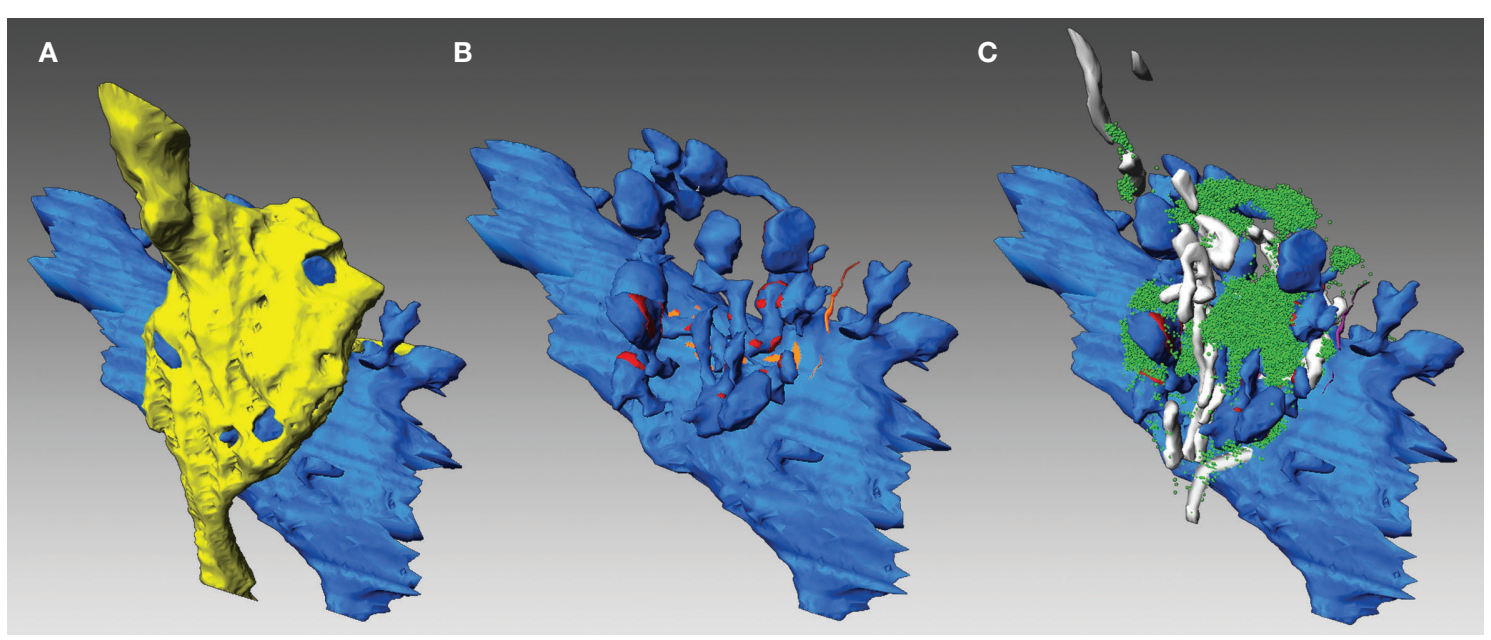

FIGURE 2 |Three-dimensional reconstructions of an adult MFB and its postsynaptic target dendrite. (A) Volume reconstructions of an en passant MFB (depicted in yellow) and its postsynaptic target dendrite (blue). Note, that the spiny excrescences were almost entirely covered by the nerve terminal. (B) Distribution of the two membrane specializations, AZs (in red) and PAs (in orange) on the postsynaptic target dendrite (blue). Note, that AZs were mainly located on the spiny excrescences, whereas PAs were exclusively found at the dendritic shaft. (C) Organization of the pool of synaptic vesicles (green dots) at an individual MFB. Here, the pool was distributed throughout the entire nerve terminal. Mitochondria (in white) formed either cluster-like arrangements or bands associated with the pool of synaptic vesicles (green dots). 
different caliber and of undefined origin (Rollenhagen et al., 2007a), a phenomenon that has not been reported yet for the MFB and other large central synapses. However, these axons never established either axo-axonic nor gap-junctional contacts with the MFBs.

When compared with other central synapses MFBs have approximately the same size as cerebellar mossy terminals $\left(69-200 \mu \mathrm{m}^{2}\right.$; Xu-Friedman and Regehr, 2003; 168-266 $\mu^{2}$; Hamori and Somogyi, 1983), but are significantly smaller than the giant calyx of Held endterminal ( $2500 \mu \mathrm{m}^{2}$; Sätzler et al., 2002) and significantly larger than thalamic (Bodor et al., 2008; Wanaverbecq et al., 2008) and neocortical synapses $\left(7-17 \mu \mathrm{m}^{2}\right.$; for review see Rollenhagen and Lübke, 2006). The size and shape of a synapse may influence in part the properties in synaptic transmission and plasticity and is also a very useful parameter for realistic numerical simulations of synaptic behavior. However, whether a direct relationship between the geometry of the terminal and the number, size and distribution of AZs and the pool of synaptic vesicles exists, remains to be investigated (see below).

\section{MEMBRANE SPECIALIZATIONS AT THE PRE- AND POSTSYNAPTIC APPOSITION ZONE}

As described for the calyx of Held-principal neuron synapses in the MNTB (Sätzler et al., 2002) and nigrothalamic terminals (Bodor et al., 2008), but not for other central synapses, two membrane specializations were found at the pre- and postsynaptic apposition zone: AZs, the sites of transmitter release and puncta adherentia (PA) that are thought to function as adhesion complexes.

\section{ACTIVE ZONES}

The number, size, and distribution of AZs within a given synapse are one of the main structural determinants in synaptic transmission and long- and short-term plasticity. By definition, an AZ is characterized by the dense accumulation of synaptic vesicles located in close proximity to the presynaptic density, the symmetric and asymmetric pre- and postsynaptic densities and the characteristic widening of the synaptic cleft. At MFBs the pre- and postsynaptic densities formed bands of electron-dense fuzzy material. At individual MFBs two types of AZs were found: the majority $(-80 \%)$ had pre- and postsynaptic densities that were frequently interrupted, appearing as perforated structures, whereas the remaining AZs showed perforations in either the pre- or postsynaptic densities or were non-perforated (Rollenhagen et al., 2007a). The average cleft width, measured in the center under the pre- and postsynaptic densities was $26.78 \pm 1.87 \mathrm{~nm}$. Interestingly, the cleft width was $17.59 \pm 2.93$ when measured at the two edges (at the appearance and disappearance of the pre- and postsynaptic densities). This difference in the means between center and the edges of AZs was significantly different $(P \leq 0.001)$ which could be of importance for realistic simulations and models of transmitter diffusion via the synaptic cleft. The distribution of AZs and PAs is illustrated in Figure 2B. Here, AZs are depicted in red and PAs in orange.

Active zones were mainly located on the spiny excrescences, whereas PAs were exclusively found on dendritic shafts. Two types of spiny excrescences could be distinguished on the postsynaptic target dendrites: So-called simple spines were contacted only by a single, but often very large AZ (Figure 3A1) or at most two AZs. In contrast, complex spines were contacted by up to eight AZs that were predominantly located opposite to spine heads (Figure 3A2). The AZs varied substantially in shape and size, forming patches, bands or ring-like structures and covered on average $10.6 \%$ of the total surface area of the spiny excrescences and therefore occupy $50 \%$ more of the total surface area than their counterparts at the calyx of Held endterminal.

On average, $\sim 20 \mathrm{AZs}$ (mean 18.30, range 7-34) with a mean surface area of $0.11 \pm 0.07 \mu \mathrm{m}^{2}$ were found in adult rats (see also Table 1). They varied substantially in shape and size as indicated by the large CV (0.64). Beside very large $\left(0.17 \mu \mathrm{m}^{2}\right)$ also very small $\left(0.07 \mu \mathrm{m}^{2}\right)$ AZs were observed (Figure 3B) that may in part contribute and explain the huge differences in synaptic efficacy and plasticity. It is noteworthy to mention that there is a dynamic change in both the number and size of AZs from development through adulthood (Rollenhagen et al., 2007a).

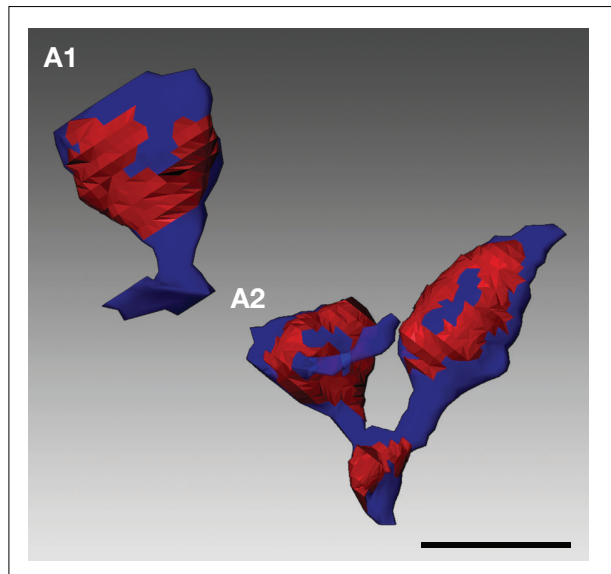

B

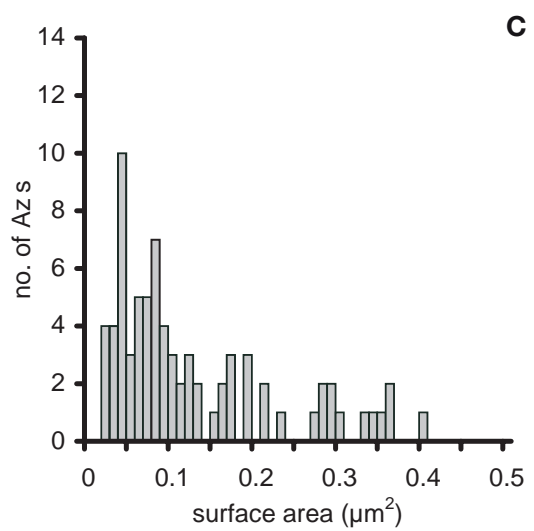

C

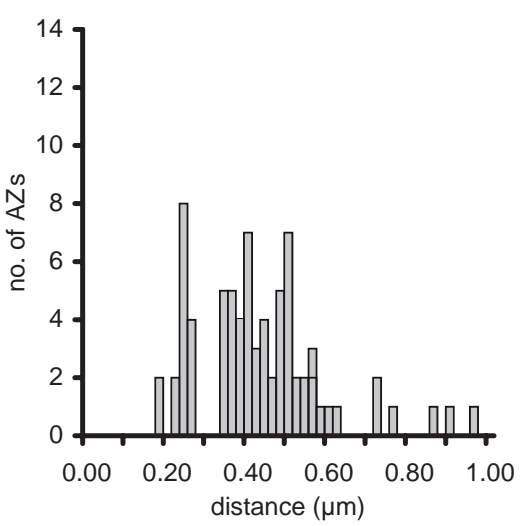

FIGURE 3 | Number, shape and location of AZs. Two representative examples of AZs (red areas) found on simple (A1) and complex (A2) spiny excrescences (blue) taken from an adult rat. Simple spines had only a single or at most two AZs whereas complex spines had up to eight AZs. Note, also the different shape and size of individual AZs that formed ring- or patch-like arrangements. Scale bar: $1 \mu \mathrm{m}$. (B) Histogram showing the distribution of surface areas of AZs for adult MFBs. (C) Histogram of the distribution of nearest-neighbor distances between centers of gravity of all AZs for adult MFBs (modified from Rollenhagen et al., 2007a). 
The size (surface area) of the AZ in MFBs was comparable with those in the calyx of Held in young rats $\left(0.10 \mu \mathrm{m}^{2}\right.$; Sätzler et al., 2002), adult deaf cats $\left(0.14 \mu \mathrm{m}^{2}\right.$; Ryugo et al., 1997), to that in climbing fiber synapses $\left(0.14 \mu \mathrm{m}^{2}\right.$; Xu-Friedman et al., 2001) and parallel fiber synapses of rat cerebellum $\left(0.13 \mu \mathrm{m}^{2}\right.$; Xu-Friedman et al., 2001). However, AZs in MFBs were larger than those in the endbulb of Held in rats $\left(0.06 \mu \mathrm{m}^{2}\right.$; Nicol and Walmsley, 2002), the calyx of Held in cats $\left(0.07 \mu \mathrm{m}^{2}\right.$; Rowland et al., 2000), GABAergic nigrothalamic terminals $\left(0.029 \mu \mathrm{m}^{2}\right.$; Bodor et al., 2008), input synapses from the nucleus reticularis thalami $\left(0.05 \mu \mathrm{m}^{2}\right)$ and anterior pretectal nucleus $\left(0.04 \mu \mathrm{m}^{2}\right)$ to the posterior thalamic nucleus (Wanaverbecq et al., 2008) and glutamatergic synapses in the hippocampal CA1 region $\left(0.06 \mu \mathrm{m}^{2}\right.$; Harris and Stevens, 1989 ; Schikorski and Stevens, 2001; Marrone et al., 2005), but significantly smaller than input synapses terminating on layer 5 pyramidal neurons $\left(0.20 \mu \mathrm{m}^{2}\right.$; Rollenhagen et al., 2007b).

Whereas the size of the AZs in MFBs was comparable to that in other central synapses, the number and density of AZs were substantially different when compared with other central synapses investigated so far (cerebellar climbing fiber synapse: 67; cerebellar mossy fiber bouton: range 113-440; nigrothalamic terminal: range 2-26; cortical synapses: range 1-3). The average number of AZs at individual MFBs was $\sim 20$, and the largest number of AZs found was 45. Thus, the MFBs contained a relatively large number of AZs (see also Chicurel and Harris, 1992) which covered on average $\sim 10 \%$ of the total surface area where the postsynaptic target apposed the presynaptic terminal. In contrast, in the calyx of Held $\sim 550$ AZs contributed only $5 \%$ to the apposition area between the pre- and postsynaptic membranes (Sätzler et al., 2002). Thus, AZs in MFBs covered a much larger fraction of the presynaptic surface area than in the calyx of Held. The density of AZs per bouton area in MFBs is important for both, synaptic strength of individual MFBs and input of multiple MFBs on the same postsynaptic CA3 pyramidal neuron, allowing convergence of $\sim 50 \mathrm{MFBs}$ on a single CA3 pyramidal neuron (Amaral and Dent, 1981).

Another functionally relevant structural factor is the spacing (nearest-neighbor relation) of individual AZs, which is one possible determinant for spillover of transmitter and hence synaptic crosstalk (Barbour and Häusser, 1997). Release sites that are close to each other suggest that glutamate spillover or synaptic crosstalk is most likely to occur at these synapses. In addition, spillover of transmitter may as a consequence desensitize receptors at neighboring postsynaptic densities, possibly resulting in a reduced synaptic efficacy (Silver et al., 1996; Ishikawa et al., 2002; for review see XuFriedman and Regehr, 2004). On the other hand, nearest neighbor AZs at extremely short distances may lead to an increase in synaptic efficacy if receptors are simultaneously activated and not saturated by the neurotransmitter (Nusser et al., 1998).

In MFBs, the nearest neighbor distance was on average $0.48 \pm 0.19 \mu \mathrm{m}$, comparable with values estimated for the cerebellar MF synapse (0.46 $\mu \mathrm{m}$; Xu-Friedman and Regehr, 2003), but different from the endbulb of Held $(0.15 \mu \mathrm{m}$; Nicol and Walmsley, 2002), the calyx of Held ( $0.59 \mu \mathrm{m}$; Sätzler et al., 2002), nigrothalamic synapses (rat: $0.17 \mu \mathrm{m}$, monkey: $0.18 \mu \mathrm{m}$; Bodor et al., 2008) and ribbon-associated $(1.66 \mu \mathrm{m})$ and ribbon-free $(1.53 \mu \mathrm{m})$ goldfish bipolar synapses (Midorikawa et al., 2007). The nearest-neighbor distances varied widely $(0.32-0.60 \mu \mathrm{m})$, as also indicated by the large standard deviation and CV. However, as $80 \%$ of the distances between neighboring $\mathrm{AZs}$ at individual MFBs were in the range between 0.32 and $0.60 \mu \mathrm{m}$ (Figure 3C), indicates that crosstalk may occur both presynaptically via diffusion of $\mathrm{Ca}^{2+}$ and postsynaptically via spillover of glutamate from the synaptic cleft, as proposed for the cerebellar MF synapses (DiGregorio et al., 2002). The differences in nearest-neighbor relationships of individual AZs over a wide range of distribution suggest that glutamate spillover and as a consequence synaptic crosstalk is differentially regulated at central synapses.

\section{PUNCTA ADHERENTIA}

The second type of membrane specializations were PAs (Figure 2B, orange structures; see Hamlyn, 1962; Honda et al., 2006). These were characterized by two parallel bands of electron dense material of approximately the same width at the pre- and postsynaptic membranes, but lacking synaptic vesicles and the characteristic broadening of the cleft typical for AZs. For PAs, the average cleft width was $20.25 \pm 1.21 \mathrm{~nm}$ in the center and $19.93 \pm 0.97 \mathrm{~nm}$ at the edges of the two densities in adult rats (see also Table 1) and are in good agreement with quantitative data reported earlier (Hamlyn, 1962). At individual MFBs the majority of PAs were organized in rows of up to 15 PAs that were exclusively found at the apposition zone of the presynaptic terminal and the dendritic shaft. The surface area of individual PAs was, on average, $0.07 \mu \mathrm{m}^{2}$, approximately half of the size of individual AZs, however, their size distribution showed a similar degree of variability (Rollenhagen et al., 2007a). An important structural difference between MFBs, the calyx of Held and nigrothalamic terminals in rat and monkey was the arrangement of the two membrane specializations. In the MFB both membrane specializations were spatially separated from each other; PAs were exclusively found at the apposition zone between the bouton and the dendritic shaft whereas AZs were located on the spiny excrescences. In contrast, in the calyx of Held PAs formed cluster-like arrangements between individual AZs that may isolate individual AZs from each other, thereby presumably preventing glutamate spillover and synaptic crosstalk. In addition, nigrothalamic terminals in rat and monkey show a centrally located network of PAs surrounded by laterally spaced AZs (Bodor et al., 2008).

\section{GLIAL COVERAGE OF MFBs}

Glial cells, in particular astrocytes and their fine processes, play an important role in synaptic function, acting as physical barriers to glutamate diffusion and mediating transmitter uptake by glutamate transporters (for review see Danbolt, 2001; Oliet et al., 2004). Strikingly, nearly all MFBs and their target structures were surrounded by astrocytic processes (Figure 1B). Thus, the synaptic complex consisting of an MFB and its postsynaptic target dendrite or spiny excrescences were physically isolated not only from the surrounding neuropil but also from neighboring synaptic complexes. Glial fingers were never observed to reach as far as to individual AZs located at the spiny excrescences or even the synaptic cleft; instead, spiny excrescences were almost completely covered by the boutons (Figure 2A). In contrast, at other central synapses, fine glial processes were observed at the pre- and postsynaptic apposition zone (Xu-Friedman and Regehr, 2003; Bodor et al., 2008), or even reached the synaptic cleft (Xu-Friedman et al., 2001; Rollenhagen et al., 2007b). As astrocytes act as physical barriers to glutamate 
diffusion and mediate transmitter uptake, e.g. by the glutamate transporters EAAT1 and EAAT2 the remote location of fine glial processes in the MFB may further enhance postsynaptic crosstalk at hippocampal MF synapses (Barbour and Häusser, 1997) whereas at the other synapses glutamate spillover and as a consequence synaptic crosstalk is not likely to occur.

Thus, two structural factors are likely to promote crosstalk at the MFB-CA3 pyramidal neuron synapse and also other synapses: (1) the short distance between adjacent AZs at spiny excrescences and (2) the absence of fine glial processes at and between AZs. Synaptic crosstalk may prolong and amplify the synaptic current, contributing to the efficacy of hippocampal MF synapses.

\section{SIZE AND ORGANIZATION OF THE POOL OF SYNAPTIC VESICLES}

The other important structural key determinant for synaptic efficacy and plasticity is the organization of the pool of synaptic vesicles, in particular, the size of the readily releasable (RRP), the recycling (RP), and the reserve pool as measured by imaging, capacitance measurements and quantal analysis studies (Rosenmund and Stevens, 1996; Schneggenburger et al., 1999; Sahara and Takahashi, 2001; Sun and Wu, 2001; Hallermann et al., 2003; Saviane and Silver, 2006; for review see Harata et al., 2001; Schneggenburger et al., 2002). However, structural data upon the composition of the three functionally defined pools of synaptic vesicles are only available for a very small number of synapses. Among the most commonly studied synapses, the striking feature is the widely different total number of synaptic vesicles in each nerve terminal, for example goldfish retinal bipolar nerve terminal: 700,000 vesicles; frog neuromuscular junction: 500,000 vesicles; calyx of Held: 700,00-180,000 vesicles; drosophila larvae neuromuscular junction: 84,000 vesicles; cerebellar mossy fiber synapses: 200,000 vesicles (Rizzoli and Betz, 2004; for review see Rizzoli and Betz, 2005).
Synaptic vesicles in individual MFBs were distributed throughout the entire terminal (Figures 1A, 2C and 4A,B) and comprised an average volume of $0.26 \mu^{3}$, corresponding to $4.2 \%$ of the total volume of the MFB. However, it is noteworthy to mention that a marked difference in the organization of the pool of synaptic vesicles was found between MFBs in postnatal day 28 (P28) and adult rats. Synaptic vesicles in young rats were evenly distributed throughout the terminal thereby completely surrounding the spiny excrescences. In adult MFBs a structural re-organization of the pool of synaptic vesicles towards a more cluster-like arrangement at individual AZs was observed (Figure 4B). These clusters are of various sizes at individual AZs and/or a row of several AZs. The distribution pattern at P28 may correspond to the functionally described 'maxipool' of synaptic vesicles described by capacitance measurements of individual MFBs (Hallermann et al., 2003). Interestingly, in both ages three different types of vesicles were found: (1) Small clear synaptic vesicles with a mean diameter of $31.20 \pm 5.20 \mathrm{~nm}$ for adult rats, ranging between $19.60 \mathrm{~nm}$ and $41.20 \mathrm{~nm}$ for individual boutons, (2) large clear vesicles (Henze et al., 2002b; $\geq 70 \mathrm{~nm}$ ) that constituted $\sim 1 \%$ of the total pool and were intermingled with the population of smaller synaptic vesicles and (3) large densecore vesicles. Dense-core vesicles are not observed in all central synapses investigated so far. Interestingly, at input synapses in layer 5 input dense core vesicles contribute with $\sim 10 \%$ to the total vesicular pool (Rollenhagen et al., 2007b) and were also seen to fuse with the AZ. It may therefore be speculated that these vesicles not only belong to the exo- and endocytosis machinery and contain various peptides and hormones (for review see Harata et al., 2006; Salio et al., 2006), but are also involved in the build-up of AZs thereby influencing the release properties and neurotransmitter trafficking at the synapse (for review see Schoch and Gundelfinger, 2006).
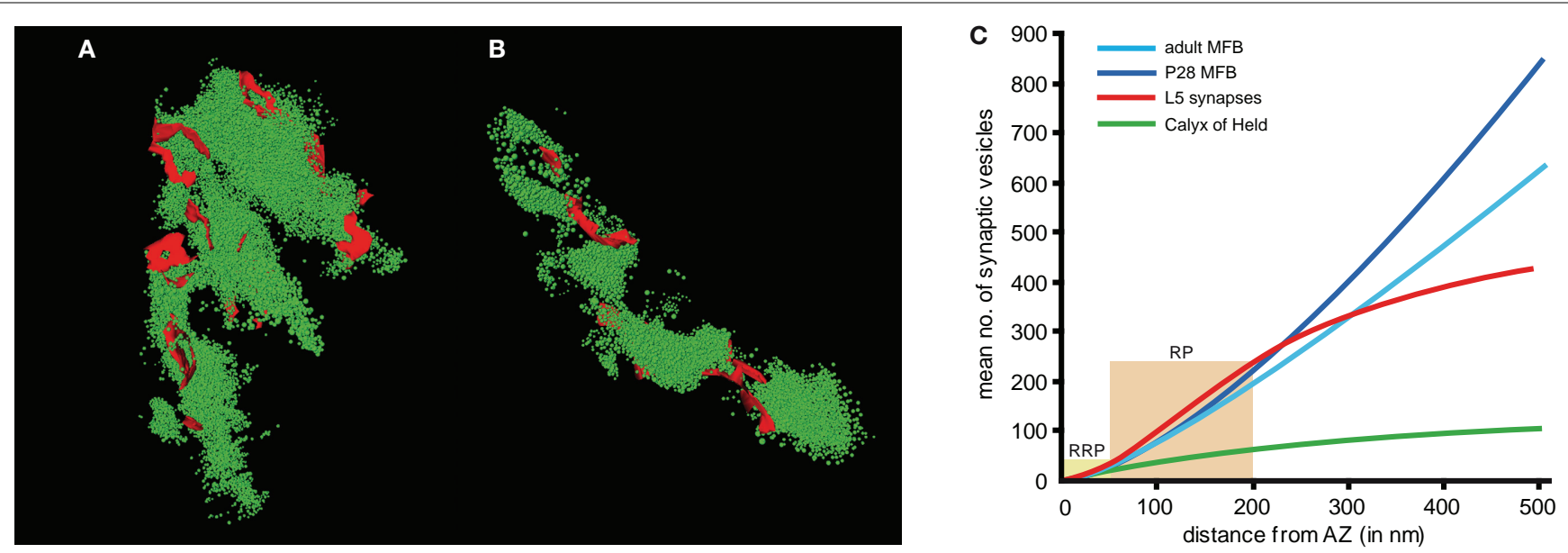

FIGURE 4 | Organization of the pool of synaptic vesicles. (A, B) Two representative examples with a large ((A) 17,138 vesicles) and smaller ((B) 7559 vesicles) total pool of synaptic vesicles (green dots) are given. (C) The graph shows the correlation for the mean number of synaptic vesicles as a function of distance from an AZ (averaged over all AZs) for P28 MFBs (light blue line), adult MFBs (dark blue line), the calyx of Held (green line) and layer 5 input synapses (red line). The light yellow boxes indicate the borders between the RRP, the RP and the reserve pool of synaptic vesicles. Note, that the slope of the curves is markedly higher in the MFBs and layer 5 input synapses when compared with the calyx of Held synapse, even though the spacing between individual AZs is comparable at these synapses. 
In order to identify subsets of synaptic vesicles as anatomical correlates for the three functionally defined pools of releasable quanta of transmitter postulated from measurements of release rates (for criteria see also Rizzoli and Betz, 2004, 2005) the RRP, the RP and the reserve pool were determined as the number of synaptic vesicles within a perimeter of $60 \mathrm{~nm}(\sim 2$ vesicle diameters), $200 \mathrm{~nm}$ ( $\sim 5$ vesicle diameters $)$ and $500 \mathrm{~nm}(\sim 15$ vesicle diameters) (Figure 4C). The number of synaptic vesicles in each of the pools was clearly correlated with the size of individual AZs (Rollenhagen et al., 2007a). Assuming a linear relationship between both measures, it was possible to define a mean vesicle density for a given perimeter. Thus, the density of synaptic vesicles at AZs in both P28 and adult MFBs (light and dark blue curves) was about two-fold higher than in the calyx of Held (green curve), but similar to values quantified for layer 5 input synapses (red curve) in the barrel field of the rat somatosensory cortex (Rollenhagen et al., 2007b).

The total pool of synaptic vesicles within individual large MFBs was on average $\sim 25,000$ (range $11,000-48,000$ ) at P28 and $\sim 16,000$ (range 7600-25,000) in adult rats (see Table 2). In contrast, the number of synaptic vesicles in small filopodia was $\sim 200-700$, two orders of magnitude smaller than in the large boutons. If the RRP is defined by $\sim 2$ vesicle diameters $(60 \mathrm{~nm})$ then the total RRP per large bouton was $\sim 1200$ vesicles (range $-350-2800$ ) in P28 and $\sim 600$ vesicles (range 370-1000) in adult rats, corresponding to $\sim 4 \%$ of the total pool of synaptic vesicles (see Table 2 ). This corresponds to $36.66 \pm 18.17$ (P28) and $41.34 \pm 20.03$ (adult) vesicles per single AZ. If the RP is defined by $\sim 5$ vesicle diameters, then the mean of the RP at individual MFBs is $\sim 5700$ (range $1800-13,100)$ vesicles $(-21 \%$ of the total pool) in P28 and $\sim 3700$ (range 1500-4700) vesicles ( $20 \%$ of the total pool) in the adult. The relative size of the RP in the MFB is in good agreement with $\mathrm{RP}$ estimates reported for other central synapses (Rizzoli and Betz, 2004, 2005). However, it has to be mentioned that the RRP

Table 2 | Synaptic vesicle pools in the adult MFB.

\begin{tabular}{|c|c|c|c|c|c|c|}
\hline \multirow[t]{3}{*}{ Bouton } & \multirow{3}{*}{$\begin{array}{l}\text { Synaptic } \\
\text { vesicles } \\
\text { Total } \\
\text { number } \\
\\
\text { No. }\end{array}$} & \multicolumn{5}{|c|}{$\begin{array}{c}\text { Size of the three different pools } \\
\text { of synaptic vesicles }\end{array}$} \\
\hline & & \multicolumn{2}{|c|}{$\begin{array}{l}\text { Putative RRP } \\
\leq 60 \mathrm{~nm}\end{array}$} & \multicolumn{2}{|c|}{$\begin{array}{c}\text { Putative RP } \\
60 \mathrm{~nm}> \\
\text { distance } \leq 200\end{array}$} & \multirow{2}{*}{$\begin{array}{l}\text { Putative } \\
\text { reserve } \\
\text { pool >200 } \\
\text { No. }\end{array}$} \\
\hline & & No. & No./AZ & No. & No./AZ & \\
\hline 1 & 13,269 & 432 & 12.71 & 2323 & 70.67 & 10,434 \\
\hline 3 & 17,138 & 599 & 42.78 & 3531 & 263.71 & 12,847 \\
\hline 4 & 25,028 & 1034 & 57.44 & 4723 & 276.83 & 19,011 \\
\hline 7 & 7559 & 367 & 52.42 & 1531 & 233.14 & 5560 \\
\hline Mean & 15,749 & 608 & 41.34 & 3720 & 211.09 & 11,963 \\
\hline CV & & 0.49 & & 0.46 & & 0.47 \\
\hline
\end{tabular}

Summary of structural parameters relevant for release that have been extracted from the detailed 3D-reconstructions. For the RRP and $R P$, two values are given: the total number of synaptic vesicles per MFB and per individual AZ. For the reserve pool, the number of synaptic vesicles per bouton is given (modified from table 1 in Rollenhagen et al., 2007a). and the RP in P28 and adult MFBs showed a large variability, as indicated by the large standard deviation and a consistently large CV of about 1.0.

Capacitance measurements at hippocampal MFBs revealed a large size of the releasable pool ('maxipool') with $\sim 1400$ vesicles released during a 30-ms pulse (Hallermann et al., 2003). However, the structural correlate of the releasable pool, although large, comprises only a small fraction of the total pool. The total pool of synaptic vesicles was $\sim 25,000$ for $\mathrm{P} 28$ and $\sim 16,000$ vesicles for adult MFBs, distributed over $\sim 30$ and $\sim 20 \mathrm{AZs}$, respectively. Thus, the total pool size of synaptic vesicles per AZ is $\sim 850$ in both ages. A very different scenario was found in the calyx of Held, where $\sim 70,000$ vesicles were distributed over $\sim 550$ release sites with $\sim 125$ synaptic vesicles per AZ. In cerebellar MF synapses, fluctuation analysis of synaptic currents suggested a total pool size of 200,000 synaptic vesicles distributed over $\sim 330$ release sites (Saviane and Silver, 2006). Thus, the number of synaptic vesicles per AZ would be $\sim 600$ at cerebellar $\mathrm{MF}$ synapses. Interestingly, the total pool per AZ in the large MFB ( 900) appears to be larger than the pool in the endbulbs emerging from filopodial extensions of the same terminal (200-700 vesicles per AZ). This may contribute to target-cell specific differences in synaptic dynamics (Maccaferri et al., 1998; Tóth et al., 2000; Pelkey and McBain, 2005; Pelkey et al., 2006).

At the calyx of Held, taking the $60 \mathrm{~nm}$ criterion, the total RRP was $\sim 10,000$ distributed over $\sim 550 \mathrm{AZs}$, equivalent to $\sim 18$ synaptic vesicles per AZ. Similarly, the putative RP was $\sim 5700$ vesicles for P28 MFBs, $\sim 3700$ vesicles for adult MFBs and $\sim 25,000$ for the calyx of Held. When estimated for individual AZs, the RP was fourfold larger in MFBs than in the calyx of Held (Figure 4C). Finally, the putative reserve pool was $\sim 18,000$ vesicles for P28 MFBs, $\sim 12,000$ for adult MFBs and 25,000 for the calyx of Held. When estimated for individual AZs the reserve pool was 10-fold larger in MFBs than in the calyx of Held (Figure 4C). The large size of the RRP, $\mathrm{RP}$, and reserve pool at each $\mathrm{AZ}$ may be a key structural correlate of presynaptic efficacy and plasticity at hippocampal MFBs (Salin et al., 1996; Geiger and Jonas, 2000).

As already mentioned above a huge variability not only in the total pool of synaptic vesicles exists, but also for the size of the readily releasable, recycling and reserve pool at different synaptic systems. In particular, the size of the RRP differs substantially between species (drosophila larvae neuromuscular junction: $\sim 300$, $0.4 \%$ of the total pool; frog neuromuscular junction: $\sim 10,000,2 \%$ of the total pool; hippocampal cultured neurons: $\sim 10,5 \%$ of the total pool; goldfish retina: $\sim 1200,0.17 \%$ of the total pool. These differences are also observed for the RP ranging from $0.6 \%$ to $20 \%$ of the total pool size. Only the size of the reserve pool with $\sim 80 \%$ of the total seems to be a constant factor between different synapses. It has to be mentioned though that the comparison of pool sizes may be inaccurate due to different experimental procedures and methods used, the state of the synapse and also the different age of the animals among species investigated.

\section{FUNCTIONAL SIGNIFICANCE OF THE STRUCTURAL COMPONENTS FOR MFB SYNAPTIC TRANSMISSION}

Both the relatively large number and distribution of release sites together with the large size of the RRP, RP, and reserve pool will have important functional implications. The large number of AZs 
together with the large RRP and RP will contribute to the high efficacy of the hippocampal MF synapse in vitro (Geiger and Jonas, 2000) and in vivo (Henze et al., 2002a; reviewed by Bischofberger et al., 2006). The high density of AZs at the pre- and postsynaptic apposition zone at the spiny excrescences is important to accommodate convergent input of multiple MFBs on the same postsynaptic CA3 pyramidal neuron (Amaral et al., 1990). The large RRP may prevent depletion during repetitive stimulation, e.g. if the animal is located in the center of the place field of a granule cell or during odor-guided behavioral tasks (reviewed by Bischofberger et al., 2006). However, even more importantly, the large size of RP and reserve pool could be used to rapidly refill the releasable pool if it has been depleted by long-lasting repetitive stimulation. If the refilling rates in MFBs were activity dependent, the large size of the RP could explain MF plasticity, e.g. the nearly 10 -fold increase in synaptic strength during frequency facilitation and post-tetanic potentiation (Salin et al., 1996). Thus, multiple structural determinants converge on the generation of a 'conditional detonator' synapse that makes the MF-CA3 pyramidal cell synapse exceptional, unique, and therefore perfectly suited for its role in the hippocampal network.

\section{PERSPECTIVE}

The quantitative structural data on the MFB provide a basis for numerical simulations and realistic models of MF transmission, which may help to constrain functional parameters that are, at present, inaccessible experimentally. However, there are still a lot of open questions to be answered in the future. At the molecular level, it has been shown using a wide spectrum of experimental methods that the basal release is regulated by presynaptic adenosine $\mathrm{A}_{1}$ receptors, that specific subtypes of metabotropic glutamate receptors strongly depress neurotransmitter release, that kainate receptors

\section{REFERENCES}

Acsády, L., Kamondi, A., Sik, A., Freund, T., and Buzsaki, G. (1998). GABAergic cells are the major postsynaptic targets of mossy fibers in the rat hippocampus. J. Neurosci. 18, 3386-3403.

Alle,H., and Geiger,J.R. (2006).Combined analog and action potential coding in hippocampal mossy fibers. Science 311, 1290-1293.

Alle, H., Roth, A., and Geiger, J. R. (2009). Energy-efficient action potentials in hippocampal mossy fibers. Science 325, 1405-1408.

Amaral, D. G., and Dent, J. A. (1981). Development of the mossy fibers of the dentate gyrus: I. A light and electron microscopic study of the mossy fibers and their expansions. J. Comp. Neurol. 195, 51-86.

Amaral, D. G., Ishizuka, N., and Claiborne, B. (1990). Neurons, numbers and the hippocampal network. Prog. Brain Res. 83, 1-11.

Barbour, B., and Häusser, M. (1997). Intersynaptic diffusion of neurotransmitter. Trends Neurosci. 20, 377-384.

Bischofberger, J., Engel, D., Frotscher, M., and Jonas, P. (2006). Timing and efficacy of transmitter release at mossy

on the other hand are involved in the increase in excitability (Kwon and Castillo, 2008), but that LTP is NMDA-receptor independent and crucially depends on the activation of P/Q and N-type $\mathrm{Ca}^{2+}$ channels. However, it is still rather unknown how these and other receptors and channels are located at the MF synapse (pre- versus postsynaptic, central versus lateral organization of receptors and their subunits at the AZ, synaptic, peri- and extrasynaptic localization) and in which density and distribution they occur. This question could be addressed using freeze fracture replica preparations combined with single- or multiple postembedding immunohistochemistry against different receptors and their subunits, and quantitative analysis in form of receptor fingerprints at individual synapses (Shigemoto et al., 1997; Masugi-Tokita et al., 2007; for review see Masugi-Tokita and Shigemoto, 2007). Furthermore, the molecular components that distinguish vesicles belonging to one of the three vesicular pools remain to be identified and which mechanisms drive the transfer from one pool to the other.

Finally, it has to be pointed out that the structural composition of synapses and the arrangement of their subelements determine the functional properties of synapses embedded in different neuronal microcircuits. Synapses are highly specialized structures that are perfectly adapted both structurally and functionally to their 'job' within a given network. Therefore, the dream of a 'model' synapse that describes the structure and function of all synapses in the brain seems to become more and more unrealistic. Thus, much more extensive work is needed to unravel the unique behavior of synapses embedded in different microcircuits of the brain.

\section{ACKNOWLEDGMENT}

The ongoing financial support by the Deutsche Forschungsgemeinschaft (grants to J.H.R.L) is gratefully acknowledged.

age-gated $\mathrm{Na}+$ channels in hippocampal mossy fiber boutons. Neuron 45, 405-417.

Frotscher, M., Jonas, P., and Sloviter, R. S. (2006). Synapses formed by normal and abnormal hippocampal mossy fibers. Cell Tissue Res. 326, 361-367.

Frotscher, M., Soriano, E., and Misgeld, U. (1994). Divergence of hippocampal mossy fibers. Synapse 16, 148-160.

Galimberti, I., Gogolla, N., Alberi, S. Santos, A. F., Muller, D., and Caroni, P. (2006). Long-term rearrangements of hippocampal mossy fiber terminal connectivity in the adult regulated by experience. Neuron 50, 749-763.

Geiger, J. R., and Jonas, P. (2000). Dynamic control of presynaptic $\mathrm{Ca}^{2+}$ inflow by fast-inactivating $\mathrm{K}^{+}$channels in hippocampal mossy fiber boutons. Neuron 28, 927-939.

Hallermann, S., Pawlu, C., Jonas, P., and Heckmann, M. (2003). A large pool of releasable vesicles in a cortical glutamatergic synapse. Proc. Natl. Acad. Sci. U.S.A. 100, 8975-8980.

Hamlyn, L. H. (1962). The fine structure of the mossy fibre endings in the hippocampus of the rabbit. J. Anat. 96, 112-120.
Hamori, J., and Somogyi, J. (1983). Differentiation of cerebellar mossy fiber synapses in the rat: a quantitative electron microscope study. J. Comp. Neurol. 220, 365-377.

Harata, N. C., Aravanis, A. M., and Tsien, R. W. (2006). Kiss-and-run and full-collapse fusion as modes of exo-endocytosis in neurosecretion. J. Neurochem. 97, 1546-1570.

Harata, N. C., Pyle, J. L., Aravanis, A. M., Mozhayeva, M., Kavalali, E. T., and Tsien, R. W. (2001). Limited numbers of recycling vesicles in small CNS nerve terminals: implications for neural signaling and vesicular cycling. Trends Neurosci. 24, 637-643.

Harris, K. M., and Stevens, J. K. (1989). Dendritic spines of CA 1 pyramidal cells in the rat hippocampus: serial electron microscopy with reference to their biophysical characteristics. J. Neurosci. 9, 2982-2997.

Henze, D. A., Wittner, L., and Buzsaki, G. (2002a). Single granule cells reliably discharge targets in the hippocampal CA3 network in vivo. Nat. Neurosci. 5, 790-795.

Henze, D. A., McMahon, D. B., Harris, K. M., and Barrionuevo, G. (2002b). 
Giant miniature EPSCs at the hippocampal mossy fiber to CA3 pyramidal cell synapse are monoquantal. $J$. Neurophysiol. 87, 15-29.

Honda, T., Sakisaka, T., Yamada, T., Kumazawa, N., Hoshino, T., Kajita, M., Kayahara, T., Ishizaki, H., TanakaOkamoto, M., Mizoguchi,A., Manabe, T., Miyoshi, J., and Takai, Y. (2006). Involvement of nectins in the formation of puncta adherentia junctions and the mossy fiber trajectory in the mouse hippocampus. Mol. Cell Neurosci. 31, 315-325.

Ishikawa, T., Sahara, Y., and Takahashi, T. (2002). A single packet of transmitter does not saturate postsynaptic glutamate receptors. Neuron 34, 613-621.

Kobayashi, K., and Poo, M. M. (2004). Spike train timing-dependent associative modification of hippocampal CA3 recurrent synapses by mossy fibers. Neuron 41, 445-454.

Kwon, H. B., and Castillo, P. E. (2008). Long-term potentiation selectively expressed by NMDA receptors at hippocampal mossy fiber synapses. Neuron 57, 108-120.

Lawrence, J. J., Grinspan, Z. M., and McBain, C. J. (2004). Quantal transmission at mossy fibre targets in the CA3 region of the rat hippocampus. J. Physiol. (Lond.) 554, 175-193.

Lisman, J.E. (1999). Relating hippocampal circuitry to function: recall of memory sequences by reciprocal dentate-CA3 interactions. Neuron 22, 233-242.

Maccaferri, G., Toth, K., and McBain, C. J. (1998). Target-specific expression of presynaptic mossy fiber plasticity. Science 279, 1368-1370.

Marrone, D. F., LeBoutillier, J.C., and Petit, T.L. (2005). Ultrastructural correlates of vesicular docking in the rat dentate gyrus. Neurosci. Lett. 378, 92-97.

Masugi-Tokita, M., and Shigemoto, R. (2007). High-resolution quantitative visualization of glutamate and GABA receptors at central synapses. Curr. Opin. Neurobiol. 17, 387-393.

Masugi-Tokita, M., Tarusawa, E., Watanabe, M., Molnar, E., Fujimoto, K., and Shigemoto, R. (2007). Number and density of AMPA receptors in individual synapses in the rat cerebellum as revealed by SDS-digested freezefracture replica labeling. J. Neurosci. 27, 2135-2144.

Midorikawa, M., Tsukamoto, Y., Berglund, K., Ishii, M., and Tachibana, M. (2007). Different roles of ribbon-associated and ribbon-free active zones in retinal bipolar cell. Nat. Neurosci. 10, 1268-1276.

Moore, K. A., Nicoll, R. A., and Schmitz, D. (2003). Adenosine gates synaptic plasticity at hippocampal mossy fiber synapses. Proc. Natl. Acad. Sci. U.S.A. 100, 14379-14402.

Nicol, M. J., and Walmsley, B. (2002). Ultrastructural basis of synaptic trans- mission between endbulbs of Held and bushy cells in the rat cochlear nucleus. J. Physiol. (Lond.) 539, 713-723.

Nicoll, R. A., and Schmitz, D. (2005). Synaptic plasticity at hippocampal mossy fibre synapses. Nat. Rev. Neurosci. 6, 863-876.

Nusser, Z., Lujan, R., Laube, G., Roberts, J. D., Molnar, E., and Somogyi, P. (1998). Cell type and pathway dependence of synaptic AMPA receptor number and variability in the hippocampus. Neuron 21, 545-559.

Oliet, S. H., Piet, R., Poulain, D. A., and Theodosis, D. T. (2004). Glial modulation of synaptic transmission: Insights from the supraoptic nucleus of the hypothalamus. Glia 47, 258-267.

Pelkey, K. A., and McBain, C. J. (2005). How to dismantle a detonator synapse. Neuron 45, 327-329.

Pelkey, K. A., Topolnik, L., Lacaille, J. C., and McBain, C. J. (2006). Compartmentalized $\mathrm{Ca}^{2+}$ channel regulation at divergent mossy-fiber release sites underlies target cell-dependent plasticity. Neuron 52, 497-510.

Rizzoli, S. O., and Betz, W. J. (2004). The structural organization of the readily releasable pool of synaptic vesicles. Science 303, 2037-2039.

Rizzoli, S. O., and Betz, W. J. (2005). Synaptic vesicle pools. Nat. Rev Neurosci. 6, 57-69.

Rizzuto, R., Bernardi, P., and Pozzan, T. (2000). Mitochondria as all-round players of the calcium game. J. Physiol. (Lond.) 529, 37-47.

Rollenhagen, A., and Lübke,J.H.R. (2006). The morphology of excitatory central synapses: from structure to function. Cell Tissue Res. 326, 221-237.

Rollenhagen, A., Sätzler, K., Rodriquez, E. P., Jonas, P., Frotscher, M., and Lübke, J. H. R. (2007a). Structural determinants of transmission at large hippocampal mossy fiber synapses. J. Neurosci. 27, 10434-10444.

Rollenhagen, A., Sätzler, K., Roth, A., and Lübke, J. H. R. (2007b). Structural determinants of transmission and plasticity of input synapses on layer 5 pyramidal neurons. Program No. 656.3. Neuroscience Meeting Planer. San Diego, CA, Society for Neuroscience, 2007. Online.

Rosenmund, C., and Stevens, C. F. (1996). Definition of the readily releasable pool of vesicles at hippocampal synapses. Neuron 16, 1197-1207.

Rowland, K. C., Irby, N. K., and Spirou, G. A. (2000). Specialized synapse-associated structures within the calyx of Held. J. Neurosci. 20, 9135-9144.

Ryugo, D. K., Pongstaporn, T., Huchton, D. M., and Niparko, J. K. (1997). Ultrastructural analysis of primary endings in deaf white cats: morphologic alterations in endbulbs of Held. J. Comp. Neurol. 385, 230-244.
Sahara, Y., and Takahashi, T. (2001). Quantal components of the excitatory postsynaptic currents at a rat central auditory synapse. J. Physiol. (Lond.) 536, 189-197.

Salin, P. A., Scanziani, M., Malenka, R. C., and Nicoll, R.A. (1996). Distinct shortterm plasticity at two excitatory synapses in the hippocampus. Proc. Natl. Acad. Sci. U.S.A. 93, 13304-13309.

Salio, C., Lossi, L., Ferrini, F., and Merighi, A. (2006). Neuropeptides as synaptic transmitters. Cell Tissue Res. 326, 583-598.

Sätzler,K., Söhl, L. F., Bollmann, J.H., Borst, J. G., Frotscher, M., Sakmann, B., and Lübke, J. H. R. (2002). Three-dimensional reconstruction of a calyx of Held and its postsynaptic principal neuron in the medial nucleus of the trapezoid body. J. Neurosci. 22, 10567-10579.

Saviane, C., and Silver, R. A. (2006). Fast vesicle reloading and a large pool sustain high bandwidth transmission at a central synapse. Nature 439, 983-987.

Schikorski, T., and Stevens, C. F. (2001) Morphological correlates of functionally defined synaptic vesicle populations. Nat. Neurosci. 4, 391-395.

Schneggenburger, R., Meyer, A. C., and Neher, E. (1999). Released fraction and total size of a pool of immediately available transmitter quanta at a calyx synapse. Neuron 23, 399-409.

Schneggenburger, R., Sakaba, T., and Neher, E. (2002). Vesicle pools and short-term synaptic depression: lessons from a large synapse. Trends Neurosci. 25, 206-212.

Schoch, S., and Gundelfinger, E. D. (2006). Molecular organization of the presynaptic active zone. Cell Tissue Res. 326 379-391.

Shigemoto, R., Kinoshita, A., Wada, E. Nomura, S., Ohishi, H., Takada, M., Flor, P. J., Neki, A., Abe, T., Nakanishi, S., and Mizunom, N. (1997). Differential presynaptic localization of metabotropic glutamate receptor subtypes in the rat hippocampus. $J$. Neurosci. 17, 7503-7522.

Sikora, M. A., Gottesman, J., and Miller, R. F. (2005). A computational model of the ribbon synapse. J. Neurosci. Methods 145, 47-61.

Silver, R. A., Colquhoun, D., Cull-Candy, S. G., and Edmonds, B. (1996). Deactivation and desensitazation of non-NMDA receptors in patches and the time course of EPSCs in rat cerebellar granule cells. J. Physiol. (Lond.) 493, 167-173.

Sun, J.Y., and Wu, L. G. (2001). Fast kinetics of exocytosis revealed by simultaneous measurements of presynaptic capacitance and postsynaptic currents at a central synapse. Neuron 30 , 171-182.

Szabadics, J., and Soltesz, I. (2009). Functional specificity of mossy fiber innervation of GABAergic cells in the hippocampus. J. Neurosci. 29, 4239-4251.

tom Dieck, S., and Brandstätter, J. H. (2006). Ribbon synapses of the retina. Cell Tissue Res. 326, 339-346.

Tóth, K., Suares, G., Lawrence, J. J., PhilipsTansey, E., and McBain, C. J. (2000). Differential mechanisms of transmission at three types of mossy fiber synapse. J. Neurosci. 20, 8279-8289.

Verstreken, P., Ly, C. V., Venken, K. J., Koh, T.W., Zhou, Y., and Bellen, H. J. (2005). Synaptic mitochondria are critical for mobilization of reserve pool vesicles at Drosophila neuromuscular junctions. Neuron 47, 365-378.

Wanaverbecq, N., Bodor, A. L., Bokor, H., Slézia, A., Lüthi, A., and Acsády, L. (2008). Constrasting the functional properties of GABAergic axon terminals with single and multiple release synapses in the thalamus. J. Neurosci. $28,11848-11861$.

Wimmer, V. C., Horstmann, H., Groh, A., and Kuner, T. (2006). Donut-like topology of synaptic vesicles with a central cluster of mitochondria wrapped into membrane protrusions: a novel structure-function module of the adult calyx of Held. J. Neurosci. 26, 109-116.

Xu-Friedman, M. A., Harris, K. M., and Regehr, W. G. (2001). Three-dimensional comparison of ultrastructural characteristics at depressing and facilitating synapses onto cerebellar Purkinje cells. J. Neurosci. 21, 6666-6672.

Xu-Friedman, M. A., and Regehr, W. G. (2003). Ultrastructural contributions to desensitization at cerebellar mossy fiber to granule cell synapses. J. Neurosci. 23, 2182-2192.

Xu-Friedman, M. A., and Regehr, W. G. (2004). Structural contributions to short-term synaptic plasticity. Physiol. Rev. 84, 69-85.

Conflict of Interest Statement: The authors declare that the research was conducted in the absence of any commercial or financial relationships that could be construed as a potential conflict of interest.

Received: 23 December 2009; paper pending published: 11 January 2010; accepted: 12 February 2010; published online: 15 March 2010.

Citation: Rollenhagen $A$ and Lübke JHR (2010) The mossy fiber bouton: the "common" or the "unique" synapse? Front. Syn. Neurosci. 2:2. doi: 10.3389/fnsyn.2010.00002

Copyright $\odot 2010$ Rollenhagen and Lübke. This is an open-access article subject to an exclusive license agreement between the authors and the Frontiers Research Foundation, which permits unrestricted use, distribution, and reproduction in any medium, provided the original authors and source are credited. 